\title{
Educação Popular em Saúde: doação de leite humano em comunidade do Rio de Janeiro, Brasil
}

Jenifer Borges Pellegrine ${ }^{(a)}$ Fabiana Ferreira Koopmans ${ }^{(b)}$

Halyne Limeira Pessanha ${ }^{(c)}$ Cleide Gonçalo Rufino(d) Helena Portes Sava de Farias ${ }^{(e)}$

\section{Introdução}

Este trabalho apresenta um relato de experiência em Educação Popular em Saúde acerca da promoção do aleitamento materno com nutrizes para a doação de leite humano (LH) em uma Unidade Básica de Saúde (UBS), localizada em uma comunidade do município do Rio de Janeiro (MRJ).

Essa experiência, promovida pela equipe dessa UBS, atuou diretamente com as nutrizes sadias da comunidade para doação de leite materno para os bancos de leite humano (BLH). Tal atuação ocorreu por meio de grupos educativos e orientações individuais na Unidade e nas visitas domiciliares, atuando sobre a realidade de cada nutriz, a fim de favorecer o aumento de doadoras de LH.

A doação de leite humano é definida como uma ação realizada por nutrizes saudáveis que apresentam excesso de produção de leite, além das necessidades normais da criança, e que se dispõe a doá-lo por livre e espontânea vontade ${ }^{1}$. Essa prática ganhou notoriedade com o surgimento dos BLHs. O primeiro BLH foi inaugurado, no Brasil, em 1943, na cidade do Rio de Janeiro, e hoje é considerado o Centro de Referência Nacional para os BLHs do país. Inicialmente sua atenção foi a crianças prematuras que não dispunham de aleitamento materno ${ }^{2}$.

Vários estudos apontam que é indiscutível o benefício advindo do leite materno, considerando-se imprescindível dispor de LH em quantidades que permitam o atendimento, nos momentos de urgência, a todos os lactentes que, por motivos clinicamente comprovados, não disponham de aleitamento ao seio, situação esta para quais os BLHs constituem uma solução ${ }^{3}$.

Na década de 1940, o início da produção de leite em pó no Brasil, aliado a outros fatores como a inserção da mulher no mercado trabalho, a soberania da publicidade comercial de leites artificiais, a própria rotina das maternidades que separavam mãe e filho após o parto, dentre outros fatores, contribuíram para a diminuição do aleitamento materno natural no país².

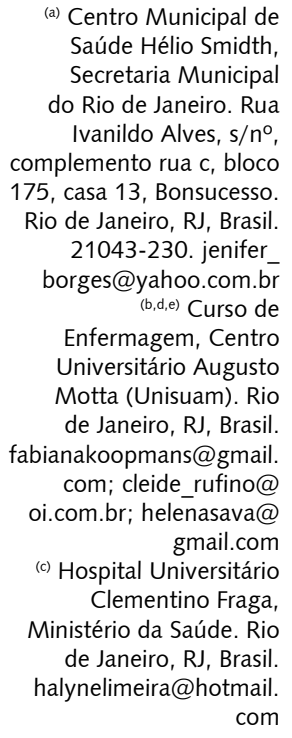

(a) Centro Municipal de Saúde Hélio Smidth, Secretaria Municipal do Rio de Janeiro. Rua Ivanildo Alves, $\mathrm{s} / \mathrm{n}^{\circ}$, complemento rua $\mathrm{c}$, bloco 175, casa 13, Bonsucesso. Rio de Janeiro, RJ, Brasil. 21043-230. jenifer

borges@yahoo.com.br $(b, d, e)$ Curso de Enfermagem, Centro Universitário Augusto Motta (Unisuam). Rio de Janeiro, RJ, Brasil. fabianakoopmans@gmail. com; cleide_rufino@ oi.com.br; helenasava@ gmail.com (c) Hospital Universitário Clementino Fraga, Ministério da Saúde. Rio de Janeiro, RJ, Brasil. halynelimeira@hotmail. com 
O desmame precoce passou a ser um importante fator no aumento do índice de mortalidade infantil, bem como de morbidade por doenças diarreicas e desnutrição².

Foi nesse contexto que, em 1988, iniciaram-se ações de incentivo ao aleitamento materno, no Brasil, pela Coordenação do Ministério da Saúde, com a criação do Programa de Incentivo ao Aleitamento Materno no início dos anos 1980, com a formulação de leis que protegem a mulher que trabalha no período da amamentação, com o combate à propaganda de leites artificiais, bicos, mamadeiras, chupetas e protetores de mamilos, inicialmente adotados no Brasil ${ }^{4}$.

Com isso a Organização Pan-Americana de Saúde (Opas) determina que o aleitamento materno é o melhor alimento que se pode oferecer para o crescimento e desenvolvimento saudável de um recémnascido ${ }^{5}$. Somado a isso, a Organização Mundial de Saúde (OMS) traz a recomendação nutricional oficial no qual o aleitamento materno deve ser exclusivo até os seis meses de idade e que continue juntamente com a alimentação complementar adequada por até dois anos de idade. Defende, ainda, a promoção de ambientes favoráveis ao aleitamento materno ${ }^{6}$.

$\mathrm{Na}$ década de 1980, a retirada e a oferta de leite foi iniciada nos BLHs, uma vez que se constatou altos índices de contaminação por leite artificial, representando $85 \%$ de óbitos infantis por desnutrição. A oferta de leite, primeiramente, era remunerada pelos BLHs de acordo com a quantidade de leite retirado, ofertavam-se cestas básicas ou serviços de saúde também como forma de pagamento devido à realidade de pobreza da época. Posteriormente, essa prática passa a ser um ato voluntário de mulheres que estão amamentando. Os BLHs aprimoram seu material de processamento do leite, bem como a capacitação de seus profissionais, idealizando o apoio ao aleitamento materno ${ }^{7}$.

Atualmente o Brasil dispõe de uma Rede de Bancos de Leite Humano, referência Latino Americana de BLH, composta de 219 BLHs e 112 Postos de Coleta de Leite Humano (PCLH), que captam e encaminham o leite doado para seus respectivos BLHs. Dentre esses, 18 BLHs e dois PCLHs estão localizados no estado do Rio de Janeiro ${ }^{8}$.

Em 1992, iniciou-se, no Brasil, a implantação da Iniciativa Hospital Amigo da Criança (Ihac), como um reforço nas ações de promoção do aleitamento materno realizadas pelo Ministério da Saúde. A Ihac é uma ação mundial que visa promover, proteger e apoiar a prática do aleitamento materno com o objetivo de diminuir o desmame precoce e suas consequências sobre a morbimortalidade infantil. Os profissionais de saúde e funcionários da unidade de saúde são mobilizados a mudar rotinas e condutas, se necessário, para diminuir o desmame precoce ${ }^{9}$.

Considerando-se que as gestações de alto risco têm seu acompanhamento em Unidades Hospitalares e que as puérperas permanecem na maternidade em um curto período após o parto, é nas UBSs que acontece a maior cobertura de pré-natal e acompanhamento da criança. Dessa forma, nas UBSs, deve-se encontrar um ambiente favorável para, em conjunto com as unidades hospitalares, desenvolver desde o início da gestação, ações de promoção ao aleitamento materno ${ }^{10}$.

Em 1999, é criada, no estado do Rio de Janeiro, a Iniciativa Unidade Básica Amiga da Amamentação (lubaam) pelo Grupo Técnico Interinstitucional de Aleitamento Materno/Paismca/SES-RJ, com o objetivo de capacitar profissionais da rede básica de saúde para a promoção, proteção e apoio ao AM, sensibilizando e mobilizando essas unidades a adotarem os "Dez Passos para o Sucesso da Amamentação", instrumento adotado pela lubaam, o qual norteia as ações de extensão da duração do período da amamentação ${ }^{11}$.

A partir dessa iniciativa, a doação de leite humano passa a ser incitada também nas UBSs, cujos profissionais deviam ser devidamente capacitados pelo lubaam, como prática de promoção do aleitamento materno. A primeira UBS a realizar essa prática foi a atualmente denominada de Clínica da Família Aloysio Augusto Novis, situada no município do Rio de Janeiro, que realiza a doação de LH para o BLH do Hospital Maternidade Herculano Pinheiro em Madureira ${ }^{12}$.

O artigo tem como objetivo relatar a experiência acerca da promoção do aleitamento materno com nutrizes para a doação de leite humano em uma UBS, localizada em uma comunidade do município do Rio de Janeiro, no período entre 2011 a 2013. 
Essa experiência, com vistas a estimular a doação de leite humano, passou por vários processos, que ocorreram desde a capacitação dos profissionais da UBS até a captação e seleção das doadoras, os grupos educativos desenvolvidos com as nutrizes, as orientações para ordenha, assim como a coleta, a conservação e o transporte de leite materno para os BLHs.

\section{Resultados e discussão}

\section{Capacitação dos profissionais e organização da UBS para captação de leite materno}

A prática de doação de leite humano nessa UBS se iniciou após a capacitação dos profissionais da Unidade pelo curso do lubaam, dentre eles: Agentes Comunitários de Saúde, enfermeiros, técnicos de enfermagem, dentistas, auxiliares de higiene dental, técnicos administrativos e médicos.

A Unidade estabeleceu vínculo com um BLH de uma maternidade de referência, fazendo articulação com o fluxo de doação com essa maternidade. Adquiriram-se materiais necessários como: maleta térmica de transporte, gelox, freezer, para estoque de leite humano, frascos de vidro, grau cirúrgico para esterilização dos frascos de vidro, touca e luvas descartáveis, formulário de controle de temperatura do freezer e de inscrição das doadoras.

Nas dependências da UBS, foi criado um local denominado "Cantinho da amamentação", idealizado para a retirada do leite quando necessário, também para orientações sobre amamentação e para, eventualmente, as mães amamentarem seus bebês, por ser um local reservado. Além disto, funcionava como um local de retirada de dúvidas sobre a prática da amamentação.

O "Cantinho da amamentação" foi criado com espaço disponível para cadeiras e poltronas, presença de armários e materiais para facilitar a coleta e ordenha do LH, seguindo todas as recomendações da Agência Nacional de Vigilância Sanitária (Anvisa), que dispõe sobre o funcionamento de BLH e PCLH para a segurança sanitária do LH ordenhado ${ }^{13}$.

Todo o processo que ocorreu junto as possíveis doadoras e nutrizes foi realizado com ênfase nas demandas das próprias nutrizes, valorizando o saber de cada uma sobre cada processo de doação de LH.

\section{Captação e seleção das doadoras para os grupos educativos}

A capacitação da equipe da UBS permitiu que os profissionais iniciassem a captação e seleção das doadoras e organizassem as ações educativas com as nutrizes. Essa captação ocorreu a todo o momento da UBS, com qualquer profissional que identificasse uma possível doadora.

Nos grupos educativos, desenvolvidos com as doadoras, foram dadas orientações sobre a coleta, a conservação e o transporte de leite materno. Partiram também de esclarecimento sobre o objetivo do $\mathrm{BLH}$, além de orientações sobre massagem e retirada do excesso de leite materno para manter o fluxo ideal ao aleitamento materno.

Assim como apontado em alguns artigos sobre doação de leite humano ${ }^{3}$, a procura pela doação ao BLH geralmente é motivada por desconforto decorrente do ingurgitamento. Em consequência disso, ocorre a doação voluntária mais constante do leite.

Ao recorrerem ao serviço de $\mathrm{BLH}$, as nutrizes recebem informações sobre a importância e o benefício proporcionado pelo seu leite e, assim, mostraram-se mais dispostas a dar continuidade à doação em prol da saúde de outros recém-nascidos ${ }^{3}$.

$\mathrm{Na}$ UBS do estudo, não foi diferente, muitas nutrizes chegavam à Unidade com esta queixa, ingurgitamento.

A figura dos Agentes Comunitários de Saúde (ACS) para a promoção da doação do leite humano foi primordial às ações da UBS, devido ao seu vínculo com a comunidade e ao seu processo de trabalho com ênfase nas visitas domiciliares. 
Os ACS apresentam grande respaldo popular e portam saberes baseados em uma forte cultura, na qual se aprende na dinâmica social. Esses atores apresentam alto poder educativo, utilizando de abordagens da Educação Popular em Saúde ${ }^{14}$.

A partir desse dado, a divulgação dessa prática de promover doação de leite humano passou a ser realizada em grupos mensais, estabelecidos na Unidade, como grupos de gestantes e nutrizes, nas visitas domiciliares, durante o teste do pezinho, vacinação, em consultas de enfermagem, odontológica e médica, em toda a comunidade adscrita a todo o momento. Passou a ser uma prática constante de toda a equipe da UBS.

\section{Doação de leite humano}

A coleta de leite ocorria tanto na Unidade, quanto no domicílio da nutriz, era realizada pela própria doadora, após a orientação de um profissional de saúde devidamente treinado. O ACS da microárea levava o frasco esterilizado e trazia-o com leite doado até o freezer da UBS para BLH da Unidade. A doação é registrada em livro de ata próprio, constando: nome da doadora, microárea, data, temperatura do freezer, quantidade de vidros doados, assinatura. Todo esse processo também seguiu as recomendações da Anvisa sobre o procedimento da coleta, a estocagem no domićlio e o preparo do frasco ${ }^{13}$.

Esse trabalho se apresentou com características básicas à amorosidade, ao vínculo e à troca entre o profissional e a doadora. Não tem como a nutriz se tornar doadora se ela não confiar no trabalho promovido na UBS. O vínculo que ela estabelece baseia-se na confiança que adquire na equipe. Essa confiança não surge só nesse momento, vem de um vínculo criado ao longo de outras experiências principalmente do acolhimento da equipe aos usuários, em todos os momentos, de variadas formas.

O profissional responsável pela captação da doadora realiza seu cadastro em formulário próprio, composto de informações pertinentes ao pré-natal, exames realizados, medicação em uso pela nutriz no período da doação, histórico de cirurgias e doenças. Semanalmente, todo o leite estocado na unidade é encaminhado ao BLH em maleta térmica com termômetro, mantendo-se a temperatura ideal com gelox, e formulário anexado de controle de temperatura.

Estudos sobre doação de leite humano enfocam que essas nutrizes vivem em lares "empobrecidos", e a realidade da UBS do estudo não poderia ser diferente, muitas doadoras não têm qualquer rendimento, ou possuem renda familiar muito abaixo das exigências atuais. Entretanto, elas se sentem motivadas a doar o excesso de um alimento essencial, em prol da vida de outros, como apontado em outro estudo ${ }^{3}$.

Isso é percebido e constatado também pelos profissionais que trabalham diretamente com a doação de leite humano da Unidade e percebido também a importância desse profissional de UBS no papel de educador em saúde. A comunicação ocorre a todo o momento, retirando dúvidas e realizando as ações conjuntamente. Para as ações ocorrerem de forma otimizada, deve-se estabelecer o vínculo e a confiança entre o profissional de saúde e a doadora de leite.

\section{Considerações finais}

A captação e doação de leite materno em USB mostram-se como uma eficaz ampliação do trabalho realizado pelos BLHs, uma vez que acompanha e orienta no próprio domicílio da nutriz o manejo da amamentação, identificando precocemente possíveis situações de risco ao aleitamento materno.

Utilizar a visita domiciliar feita por profissional capacitado, entendendo a importância de valorizar o saber e as dúvidas das nutrizes durante a coleta de leite materno favorece a promoção ao aleitamento materno, dentre tantas já desempenhadas desde a criação dos BLHs.

Partindo de alguns estudos, que apontam que as ações educativas e de acolhimento nos serviços de pré-natal, em maternidades e berçários, realizadas com qualidade e humanização, são fundamentais para a captação de doadoras de leite humano 3,15 , permite demonstrar que é possível também realizar a promoção de doação de leite humano nas UBSs, constituindo, assim, uma prática de Educação Popular em Saúde. 
Muitas mulheres multíparas que experimentam, pela primeira vez, o ato de doação permitem supor que talvez poderiam ter sido doadoras nas experiências anteriores. A não-ocorrência do comportamento de doação nas gestações pregressas pode ter sofrido influência de aspectos pessoais (desinformação, falta de iniciativa), bem como de falhas no processo de apoio institucional, como o repasse de informações no sentido de esclarecer eventuais dúvidas sobre doação de leite humano ${ }^{15}$.

Um dos pontos principais deste trabalho passa pelo vínculo que o profissional deve estabelecer com a nutriz. As doadoras de leite humano são captadas nos grupos de gestantes, nas orientações às puérperas, nas salas de espera, por toda a equipe, a todo o momento na UBS e também fora dela, nas visitas domiciliares.

As orientações, no momento de captação e também no momento de doação de leite humano, com trocas entre os profissionais e as nutrizes, foram fundamentais para que essa ação acontecesse de forma satisfatória para todos. Para as nutrizes, percebe-se uma maior aproximação e confiança no ato de doar seu leite para os BLHs. Para a UBS, essas ações realizadas por toda a equipe funcionou positivamente, pois assim que aumentava o vínculo das gestantes nas consultas e nos grupos educativos, aumentava também a doação de leite humano.

$O$ incentivo ao aleitamento materno atualmente conta com diversas iniciativas identificadas em vários níveis de atenção em saúde. O estímulo à doação de leite humano, promovido pela UBS, e a forma como foram realizadas as ações, utilizando aspectos como amorosidade, favorecimento de vínculo, valorização do saber popular, garantiram um maior impacto na promoção do aleitamento materno na unidade em questão. Percebeu-se um aumento de doadoras de leite humano e na produção de leite humano doado.

\section{Colaboradores}

Jenifer Borges Pelegrine e Fabiana Ferreira Koopmans participaram, igualmente, da elaboração do artigo, de sua discussão e redação e da revisão do texto. Halyne Limeira Pessanha, Helena Portes Sava de Farias e Cleide Gonçalo Rufino participaram da revisão bibliográfica, de discussões e revisão do manuscrito.

\section{Referências}

1. Brasil. Ministério da Saúde. Banco de leite humano: funcionamento, prevenção e controle de riscos. Brasília, DF: MS; 2008.

2. Vinagre RD, Diniz EMA, Vaz AC. Leite humano: um pouco de sua história. São Paulo: Atheneu; 2001.

3. Galvão MTG, Vasconcelos SG, Paiva SS. Mulheres doadoras de leite humano. Acta Paul Enferm. 2006;19(2):157-61.

4. Toma TS, Rea MF. Benefícios da amamentação para a saúde da mulher e da criança: um ensaio sobre as evidências. Cad Saude Publica. 2008;24 Supl. 2:S235-46.

5. Organização Pan-Americana de Saúde. Amamentação: informações institucionais, técnicas, projetos, publicações e serviços [Internet]. Brasília (DF): OPAS; 2003. [acesso 2011 Jun 17]. Disponível em: http://www.opas.org.br/sistema/fotos/amamentar.pdf

6. World Health Organization. Department of Nutricion for Health and Development. Department of Child and Adolescent Health and Development. WHO \NHD 01.08. The optimal duration of exclusive breastfeeding: a systematic review. Geneva: WHO; 2002.

7. Ministério da Saúde. Manual técnico para Banco de Leite Humano. Brasília, DF: MS; 2008.

8. Ministério da Saúde. Bancos de Leite Humano no Brasil - rede de Bancos de Leite Humano. Brasília, DF: MS; 2013. 
9. Araújo MFM, Otto AFN, Schimitz BAS. Primeira avaliação do cumprimento dos "Dez passos para o sucesso do aleitamento" nos Hospitais Amigos da Criança do Brasil. Rev Bras Saude Mat Infant. 2003;3(4):411-9.

10. Oliveira MIC, Camacho LAB, Souza OEO. Promoção, proteção e apoio à amamentação na atenção primária à saúde no Estado do Rio de Janeiro, Brasil: uma política de saúde pública baseada em evidência. Cad Saude Publica. 2005;21(6):1901-10.

11. Secretaria de Saúde do Rio de Janeiro. Manual de capacitação de multiplicadores na Iniciativa Unidade Amiga da Amamentação (IUBAAM). Rio de Janeiro: Secretaria de Saúde de Rio de Janeiro; 2006.

12. Ministério da Saúde. Rede brasileira de Bancos de Leite Humano - notícias. Brasília, DF: MS; 2012.

13. Resolução RDC $n^{\circ} 171$, de 4 de setembro de 2006. Dispõe sobre o regulamento técnico para o funcionamento de Bancos de Leite Humano. Diário Oficial da União. 2006.

14. Vasconcelos EM. Educação popular nos serviços de saúde. 3a ed. São Paulo: Hucitec; 1997.

15. Alencar LCE, Seidl EMF. Doação de leite humano: experiência de mulheres doadoras. Rev Saude Publica. 2009;43(1):70-7. 
Este artigo apresenta uma experiência de Educação Popular em Saúde para a doação de leite humano. Tem o objetivo de relatar a experiência acerca da promoção do aleitamento materno com nutrizes, para a doação de leite em uma Unidade Básica de Saúde (UBS), localizada no município do Rio de Janeiro. Apresenta todo o processo desde a capacitação dos profissionais da Unidade, os grupos educativos desenvolvidos com as nutrizes, a captação e seleção da doadora, assim como a coleta, a conservação, o transporte do leite doado até os bancos de leite humano. Todo esse processo ocorreu com valorização dos princípios da Educação Popular em Saúde, como amorosidade e valorização do saber da doadora. Dessa forma, percebeu-se que quanto mais se aumentava o vínculo das nutrizes com os profissionais da UBS, aumentava o número de doadoras e também de leite humano doado.

Palavras-chave: Leite humano. Aleitamento materno. Educação em Saúde.

\section{Popular Education in Health: human milk donation in a community in Rio de Janeiro, Brazil}

This paper presents an experience related to Popular Education in Health, focused on promoting donation of breast milk. Its main objective is to describe the experience of promoting breastfeeding with nursing mothers, aiming at breast milk donation at a Primary Care Unit located in Rio de Janeiro, Brazil. It reports the entire process: the training offered to the Unit's professionals, the educational groups held with the nursing mothers, the selection of donors, and the collection, preservation, and transportation of donated breast milk to the Human Milk Bank. The whole process respected the guiding principles of popular education in health, such as lovingness and respect for the donors' knowledge. Thus, it was possible to perceive that the stronger the bond between nursing mothers and the Unit's professionals, the higher the number of breast milk donors and the amount of donated milk.

Keywords: Human milk. Breastfeeding. Health Education.

\section{Educación Popular en Salud: donación de leche humana en comunidad de Río de Janeiro, Brasil}

Este artículo presenta una experiencia de Educación Popular en Salud para donación de leche humana. Su objetivo es la experiencia sobre la promoción de la lactancia materna con nutrices, para donación de leche en una Unidad Básica de Salud (UBS), localizada en Río de Janeiro, Brasil. Presenta todo el proceso, desde la capacitación de los profesionales de la Unidad, los grupos educativos desarrollados con las nutrices, la captación y selección de la donadora y la colecta, conservación y transporte de la leche donada hasta los bancos de leche. El proceso valorizó los principios de la Educación Popular en Salud, como amor y valorización del saber de la donadora. Así, se percibió que cuanto mayor era el vínculo de las nutrices con los profesionales de la UBS, mas aumentaban las donadoras y la leche humana donada.

Palabras clave: Leche humana. Lactancia materna. Educación en Salud.

Recebido em 11/06/2013. Aprovado em 08/12/2013. 
